\title{
A Family of Well-Clear Boundary Models for the Integration of UAS in the NAS
}

\author{
César A. Muñoz* Anthony Narkawicz James Chamberlain María Consiglio \\ Jason Upchurch \\ NASA Langley Research Center, Hampton, VA, 23881, US
}

\begin{abstract}
The FAA-sponsored Sense and Avoid Workshop for Unmanned Aircraft Systems (UAS) defines the concept of sense and avoid for remote pilots as "the capability of a UAS to remain well clear from and avoid collisions with other airborne traffic." Hence, a rigorous definition of well clear is fundamental to any separation assurance concept for the integration of UAS into civil airspace. This paper presents a family of well-clear boundary models based on the TCAS II Resolution Advisory logic. For these models, algorithms that predict well-clear violations along aircraft current trajectories are provided. These algorithms are analogous to conflict detection algorithms but instead of predicting loss of separation, they predict whether well-clear violations will occur during a given lookahead time interval. Analytical techniques are used to study the properties and relationships satisfied by the models.
\end{abstract}

\section{Nomenclature}

D Horizontal distance parameter

DTHR, TTHR Horizontal distance and time thresholds

$d_{\text {cpa }}$

Distance at closest point of approach

$\epsilon$

Numerical parameter whose value is \pm 1

$\mathbf{s}, \mathbf{v}$

$s_{z}, v_{z}$

$t_{\text {var }}$

$\tau, \tau_{\bmod }$

$t_{\text {cpa }}$

$t_{\text {ep }}$

Two-dimensional aircraft state, i.e., position and velocity

Vertical aircraft state, i.e., altitude and vertical speed

Time variable

TCAS II tau and modified tau

Time to closest point of approach

Time to entry point

Time to co-altitude

ZTHR, TCOA

Subscripts

$o, i$

$x, y, z$

Vertical distance and time thresholds

Ownship and intruder information of a position or velocity vector

Acronyms

CAT

Northern, eastern, and altitude component of a position or velocity vector

NAS

NMAC

RA

SAA

SST

SSV

TCAS

TCPA

Collision Avoidance Threshold

National Airspace System

Near Mid-Air Collision

Resolution Advisory

Sense and Avoid

Self-Separation Threshold

Self-Separation Volume

Traffic Alerting and Collision Avoidance System

Time to Closest Point of Approach

UAS Unmanned Aircraft Systems

\footnotetext{
${ }^{*}$ Corresponding Author. Email: cesar.a.munoz@nasa.gov. AIAA Member.
} 


\section{Introduction}

One of the major challenges of integrating Unmanned Aircraft Systems (UAS) into the airspace system is the lack of an on-board pilot to comply with the legal requirement that pilots see and avoid other aircraft in their vicinity. To address this challenge, the final report of the FAA-sponsored Sense and Avoid (SAA) Workshop for Unmanned Aircraft Systems [2] defines the concept of sense and avoid for remote UAS pilots as "the capability of a UAS to remain well clear from and avoid collisions with other airborne traffic." Under this definition, a rigorous definition of well clear becomes fundamental to any sense and avoid concept that involves UAS.

NASA's Unmanned Aircraft Systems Integration in the National Airspace System (UAS in the NAS) project aims at conducting research towards the integration of civil UAS into non-segregated airspace operations. As part of this project, NASA has developed a sense and avoid concept for UAS that extends the concept outlined by the SAA Workshop [1]. The NASA concept includes a volume, namely the Self Separation Volume (SSV), located between the Collision Avoidance Threshold (CAT), defined by collision avoidance systems, and the Self-Separation Threshold (SST), defined by self-separation systems [2]. The SSV represents a well-clear boundary where aircraft inside the SSV are considered to be in well-clear violation. This volume is intended to be large enough to avoid safety concerns for controllers and see-and-avoid pilots, but small enough to avoid disruptions to traffic flow. A key characteristic of NASA's concept is that the SSV is a conservative extension of the CAT defined by the Traffic Alerting and Collision Avoidance System (TCAS).

TCAS is a family of airborne devices that are designed to reduce the risk of mid-air collisions between aircraft equipped with operating transponders [10]. TCAS II, the current generation of TCAS devices, is mandated in the US for aircraft with greater than 30 seats or a maximum takeoff weight greater than 33,000 pounds. Although it is not required, TCAS II is also installed on many turbine-powered general aviation aircraft. Version 7.0 is the current operationally-mandated version of TCAS II, and Version 7.1 has been standardized [8]. In contrast to TCAS I, the first generation of TCAS devices, TCAS II provides resolution advisories (RAs). RAs are visual and vocalized alerts that direct pilots to maintain or increase vertical separation with intruders that are considered collision threats. TCAS II resolution advisories can be corrective or preventive depending on whether the pilot is expected to change or maintain the aircraft's current vertical speed. Corrective RAs are particularly disruptive to the air traffic system since they may cause drastic evasive maneuvers. For this reason, they are intended as a last resort maneuver when all other means of separation have failed.

The core of the TCAS II RA logic is a test that checks distance and time variables for the horizontal and vertical dimensions against a set of pre-defined threshold values. To ensure interoperability between NASA's SSA concept and TCAS, the mathematical definition of the volume SSV is based on the TCAS II Resolution Advisory Logic [5]. The definition of SSV follows the same logic, but uses different thresholds that conservatively extends the collision avoidance threshold provided by TCAS.

This paper generalizes the definitions of the well-clear boundary provided in [5]. In particular, it presents a family of well-clear boundary models based on different, but related, time variables. Formal techniques are used to study properties of these models such as symmetry, local-convexity, and inclusion. For each one of these models, an algorithm is provided that computes the time interval during which aircraft are not well clear assuming that they continue along their current trajectories. These detection algorithms assume the availability of accurate state vector information for the two aircraft. These algorithms completely and correctly characterize all encounter geometries that will cause a well-clear violation within a given lookahead time, assuming linear aircraft trajectories.

The formal development presented in this paper is part of the NASA's Airborne Coordinated Resolution and Detection (ACCoRD) mathematical framework, which is electronically available from http://shemesh .

larc.nasa.gov/people/cam/ACCoRD. All theorems in this paper have been formally verified in the Prototype Verification System (PVS) [7], an automated theorem prover. 


\section{Distance and Time Variables}

Distance and time variables are important elements of any separation assurance concept. These variables are functions over the aircraft current states which are compared against distance and time thresholds. Many conflict detection and resolution systems rely on the time of closest point of approach and the distance at that time as their main time and distance variables [4]. This section describes some additional distance and time variables that are particularly relevant to the definition of a well-clear boundary model.

This paper assumes that accurate aircraft surveillance information is available as horizontal and vertical components in a three-dimensional (3-D) airspace. Letters in bold-face denote two-dimensional (2-D) vectors. Vector operations such as addition, subtraction, scalar multiplication, dot product, i.e., $\mathbf{s} \cdot \mathbf{v} \equiv$ $s_{x} v_{x}+s_{y} v_{y}$, the square of a vector, i.e., $\mathbf{s}^{2} \equiv \mathbf{s} \cdot \mathbf{s}$, and the norm of a vector, i.e., $\|\mathbf{s}\| \equiv \sqrt{\mathbf{s}^{2}}$, are defined in a 2-D Euclidean geometry. Furthermore, the expression $\mathbf{v}^{\perp}$ denotes the 2-D right perpendicular of $\mathbf{v}$, i.e., $\mathbf{v}^{\perp} \equiv\left(v_{y},-v_{x}\right)$, and $\mathbf{0}$ denotes the 2 -D vector whose components are 0 , i.e., $\mathbf{0} \equiv(0,0)$.

The mathematical models presented in this paper consider two aircraft referred to as the ownship and the intruder aircraft. For the ownship, the current horizontal position and velocity are denoted $\mathbf{s}_{o}$ and $\mathbf{v}_{o}$, respectively. Its altitude and vertical speed are denoted $s_{o z}$ and $v_{o z}$, respectively. Similarly, the horizontal position and velocity of the intruder aircraft are denoted $\mathbf{s}_{i}$ and $\mathbf{v}_{i}$, respectively, and its vertical altitude and speed are denoted $s_{i z}$ and $v_{i z}$, respectively. As it simplifies the mathematical development, this paper uses a relative coordinate system where the intruder is static at the center of the coordinate system. In this relative system, $\mathbf{s}=\mathbf{s}_{o}-\mathbf{s}_{i}$ and $\mathbf{v}=\mathbf{v}_{o}-\mathbf{v}_{i}$ represent the horizontal relative position and velocity of the aircraft, respectively. Furthermore, $s_{z}=s_{o z}-s_{i z}$ and $v_{z}=v_{o z}-v_{i z}$ represent the vertical relative position and speed of the aircraft, respectively.

Assuming constant relative horizontal velocity $\mathbf{v}$, the horizontal range between the aircraft at any time $t$ is given by

$$
r(t) \equiv\|\mathbf{s}+t \mathbf{v}\|=\sqrt{\mathbf{s}^{2}+2 t(\mathbf{s} \cdot \mathbf{v})+t^{2} \mathbf{v}^{2}} .
$$

The time of horizontal closest point of approach, denoted $t_{\text {cpa }}$, is the time $t$ that satisfies $\dot{r}(t)=0$, i.e., $t=-\frac{\mathbf{s} \cdot \mathbf{v}}{\mathbf{v}^{2}}$. The dot product $\mathbf{s} \cdot \mathbf{v}$ characterizes whether the aircraft are horizontally diverging, i.e., $\mathbf{s} \cdot \mathbf{v}>0$, or horizontally converging, i.e., $\mathbf{s} \cdot \mathbf{v}<0$. By convention, $t_{\mathrm{cpa}}$ is defined as 0 when $\mathbf{v}=\mathbf{0}$. Hence, $t_{\mathrm{cpa}}$ is formally defined as

$$
t_{\mathrm{cpa}}(\mathbf{s}, \mathbf{v}) \equiv \begin{cases}-\frac{\mathbf{s} \cdot \mathbf{v}}{\mathbf{v}^{2}} & \text { if } \mathbf{v} \neq \mathbf{0} \\ 0 & \text { otherwise }\end{cases}
$$

It is noted that $t_{\mathrm{cpa}}(\mathbf{s}, \mathbf{v})>0$ when the aircraft are horizontally converging, $t_{\mathrm{cpa}}(\mathbf{s}, \mathbf{v})<0$ when the aircraft are horizontally diverging, and $t_{\mathrm{cpa}}(\mathbf{s}, \mathbf{v})=0$ when the aircraft are neither converging or diverging. The distance at time of closest point of approach is defined as

$$
d_{\mathrm{cpa}}(\mathbf{s}, \mathbf{v}) \equiv r\left(t_{\mathrm{cpa}}(\mathbf{s}, \mathbf{v})\right)=\left\|\mathbf{s}+t_{\mathrm{cpa}}(\mathbf{s}, \mathbf{v}) \mathbf{v}\right\| .
$$

In the vertical dimension, assuming constant relative vertical speed, the relative altitude between the aircraft at any time $t$ is given by

$$
r_{z}(t) \equiv\left|s_{z}+t v_{z}\right| .
$$

The time to co-altitude $t_{\text {coa }}$ is the time $t$ that satisfies $r_{z}(t)=0$, i.e, $t=\frac{s_{z}}{v_{z}}$. Similar to the horizontal case, the product $s_{z} v_{z}$ characterizes whether the aircraft are vertically diverging, i.e., $s_{z} v_{z}>0$, or vertically converging, i.e., $s_{z} v_{z}<0$. This paper defines time to co-altitude as -1 when the aircraft are not vertically converging. Therefore,

$$
t_{\text {coa }}\left(s_{z}, v_{z}\right) \equiv \begin{cases}-\frac{s_{z}}{v_{z}} & \text { if } s_{z} v_{z}<0, \\ -1 & \text { otherwise. }\end{cases}
$$

Formula (5) is well defined since $s_{z} v_{z}<0$ implies that $v_{z} \neq 0$.

\section{II.A. Horizontal Time Variables}

A (horizontal) time variable is a function that maps a relative horizontal position and velocity into a real number. This real number is negative when the aircraft are horizontally diverging. When the real number is non-negative, this number represents a time that, in a separation assurance logic, is intended to be compared 
against a time threshold. In this paper, the time threshold is called TTHR. An example of a time variable that is used in conflict detection logics is $t_{\text {cpa }}$ [4].

The time variable used in earlier versions of the TCAS detection logic is called tau, denoted $\tau$ [8]. Tau estimates $t_{\text {cpa }}$, but is less demanding on sensor and surveillance technology than $t_{\text {cpa }}$. Indeed, $\tau$ is simply defined as range over closure rate, where closure rate is the negative of the range rate, i.e., $\tau=-\frac{r(0)}{\dot{r}(0)}=$ $-\frac{\|\mathbf{s}\|}{\|\mathbf{s} \cdot \mathbf{s}\|}=-\frac{\mathbf{s}^{2}}{\mathbf{s} \cdot \mathbf{v}}$. This paper defines $\tau$ as -1 when the aircraft are not horizontally converging. Formally,

$$
\tau(\mathbf{s}, \mathbf{v}) \equiv \begin{cases}-\frac{\mathbf{s}^{2}}{\mathbf{s} \cdot \mathbf{v}} & \text { if } \mathbf{s} \cdot \mathbf{v}<0 \\ -1 & \text { otherwise }\end{cases}
$$

For a limited number of scenarios, the values of $\tau$ and $t_{\text {cpa }}$ coincide. However, in most scenarios, the value of $\tau$ tends toward infinity as the aircraft approach the closest point of approach. In general, $\tau$ is a good approximation of $t_{\text {cpa }}$, but only for large values. For that reason, TCAS II uses a modified variant of $\tau$ called modified tau, denoted $\tau_{\bmod }$ [8]. Modified tau provides a better estimation of $t_{\mathrm{cpa}}$ and has a more regular behavior than $\tau$ in the proximity of the closest point of approach. In [3], modified tau is defined such that $\tau_{\text {mod }}=-\frac{r(0)^{2}-\mathrm{DTHR}^{2}}{\dot{r}(0)}=\frac{\mathrm{DTHR}^{2}-\mathbf{s}^{2}}{\mathbf{s} \cdot \mathbf{v}}$. Similar to $\tau, \tau_{\bmod }$ is defined as -1 when the aircraft are not horizontally converging, i.e.,

$$
\tau_{\text {mod }}(\mathbf{s}, \mathbf{v}) \equiv \begin{cases}\frac{\mathrm{DTHR}^{2}-\mathbf{s}^{2}}{\mathbf{s} \cdot \mathbf{v}} & \text { if } \mathbf{s} \cdot \mathbf{v}<0 \\ -1 & \text { otherwise }\end{cases}
$$

The definition of $\tau_{\text {mod }}$ in Formula (7) depends on DTHR, which is a horizontal distance threshold. This threshold is called DMOD in the TCAS II RA logic and its actual value depends on a sensitivity level based on the ownship's altitude [8].

In [6], a time variable called time to entry point, denoted $t_{\mathrm{ep}}$, is proposed. Time to entry point is defined as the time to loss of horizontal separation with respect to DTHR assuming straight-line aircraft trajectories. Similar to $t_{\mathrm{cpa}}, t_{\mathrm{ep}}$ decreases linearly over time. Time to entry point is formally defined as

$$
t_{\mathrm{ep}}(\mathbf{s}, \mathbf{v}) \equiv \begin{cases}\Theta(\mathbf{s}, \mathbf{v}, \text { DTHR },-1) & \text { if } \mathbf{s} \cdot \mathbf{v}<0 \text { and } \Delta(\mathbf{s}, \mathbf{v}, \text { DTHR }) \geq 0 \\ -1 & \text { otherwise }\end{cases}
$$

where

$$
\begin{aligned}
\Theta(\mathbf{s}, \mathbf{v}, D, \epsilon) & \equiv \frac{-\mathbf{s} \cdot \mathbf{v}+\epsilon \sqrt{\Delta(\mathbf{s}, \mathbf{v}, D)}}{\mathbf{v}^{2}} \\
\Delta(\mathbf{s}, \mathbf{v}, D) & \equiv D^{2} \mathbf{v}^{2}-\left(\mathbf{s} \cdot \mathbf{v}^{\perp}\right)^{2}
\end{aligned}
$$

The function $\Theta$ is only defined when $\mathbf{v} \neq \mathbf{0}$ and $\Delta(\mathbf{s}, \mathbf{v}, D) \geq 0$. In this case, it computes the times when the aircraft will lose separation, if $\epsilon=-1$, or regain separation, if $\epsilon=1$, with respect to $D$. When the aircraft are not horizontally converging or $\Delta(\mathbf{s}, \mathbf{v}$, DTHR $)<0$, time to entry point is defined as -1 . Formula $(8)$ is well defined since the condition $\mathbf{s} \cdot \mathbf{v}<0$ guarantees that $\mathbf{v} \neq \mathbf{0}$.

\section{II.B. Properties of Horizontal Time Variables}

A useful property of a time variable is symmetry. A time variable $t_{\mathrm{var}}$ is said to be symmetric if and only for all $\mathbf{s}, \mathbf{v}$

$$
t_{\mathrm{var}}(\mathbf{s}, \mathbf{v})=t_{\mathrm{var}}(-\mathbf{s},-\mathbf{v})
$$

Symmetry guarantees that in a pairwise scenario both the ownship and intruder aircraft computes the same value for the time variable. Hence, checking a symmetric time variable against a given time threshold returns the same Boolean value for both aircraft.

Theorem 1. The time variables $t_{c p a}, \tau, \tau_{m o d}$, and $t_{e p}$ are symmetric. 
It is possible to define time variables that are not symmetric. For instance, a time variable that computes the first time when the intruder aircraft enters an elliptical area aligned to the ownship trajectory is not symmetric for every scenario. However, any time variable can be transformed into a symmetric one by using min and max operators. For instance, the time variables $\min \left(t_{\mathrm{var}}(\mathbf{s}, \mathbf{v}), t_{\mathrm{var}}(-\mathbf{s},-\mathbf{v})\right)$ and $\max \left(t_{\mathrm{var}}(\mathbf{s}, \mathbf{v}), t_{\mathrm{var}}(-\mathbf{s},-\mathbf{v})\right)$ are symmetric for any time variable $t_{\mathrm{var}}$.

Figure 1 shows a graph of time vs. $\tau, t_{\mathrm{ep}}, \tau_{\mathrm{mod}}$, and $t_{\mathrm{cpa}}$ for an initial scenario where the ownship and intruder aircraft are located at (0 nmi, $-3.25 \mathrm{nmi})$ and $(-6.25 \mathrm{nmi}, 0.25 \mathrm{nmi})$, respectively, flying at co-altitude, the ownship ground speed is $150 \mathrm{kts}$, heading $53^{\circ}$, and the intruder ground speed is $350 \mathrm{kts}$, heading $90^{\circ} .{ }^{\mathrm{a}}$ In this scenario, the distance threshold DTHR used in the definition of $\tau_{\bmod }$ and $t_{\mathrm{ep}}$ is $1 \mathrm{nmi}$. This scenario illustrates that while $t_{\mathrm{ep}}, \tau_{\text {mod }}$, and $t_{\text {cpa }}$ decrease over time, the time variable $\tau$ decreases up to some point, but then it abruptly increases in the vicinity of the closest point of approach. Moreover, when these time variables are checked against a time threshold TTHR, represented by the horizontal line at 30 seconds, the time variable $t_{\mathrm{ep}}$ crosses the time threshold first, followed by $\tau_{\text {mod }}, t_{\text {cpa }}$, and $\tau$, in that order. Interestingly, this property holds for any converging scenario and any choice of threshold values.

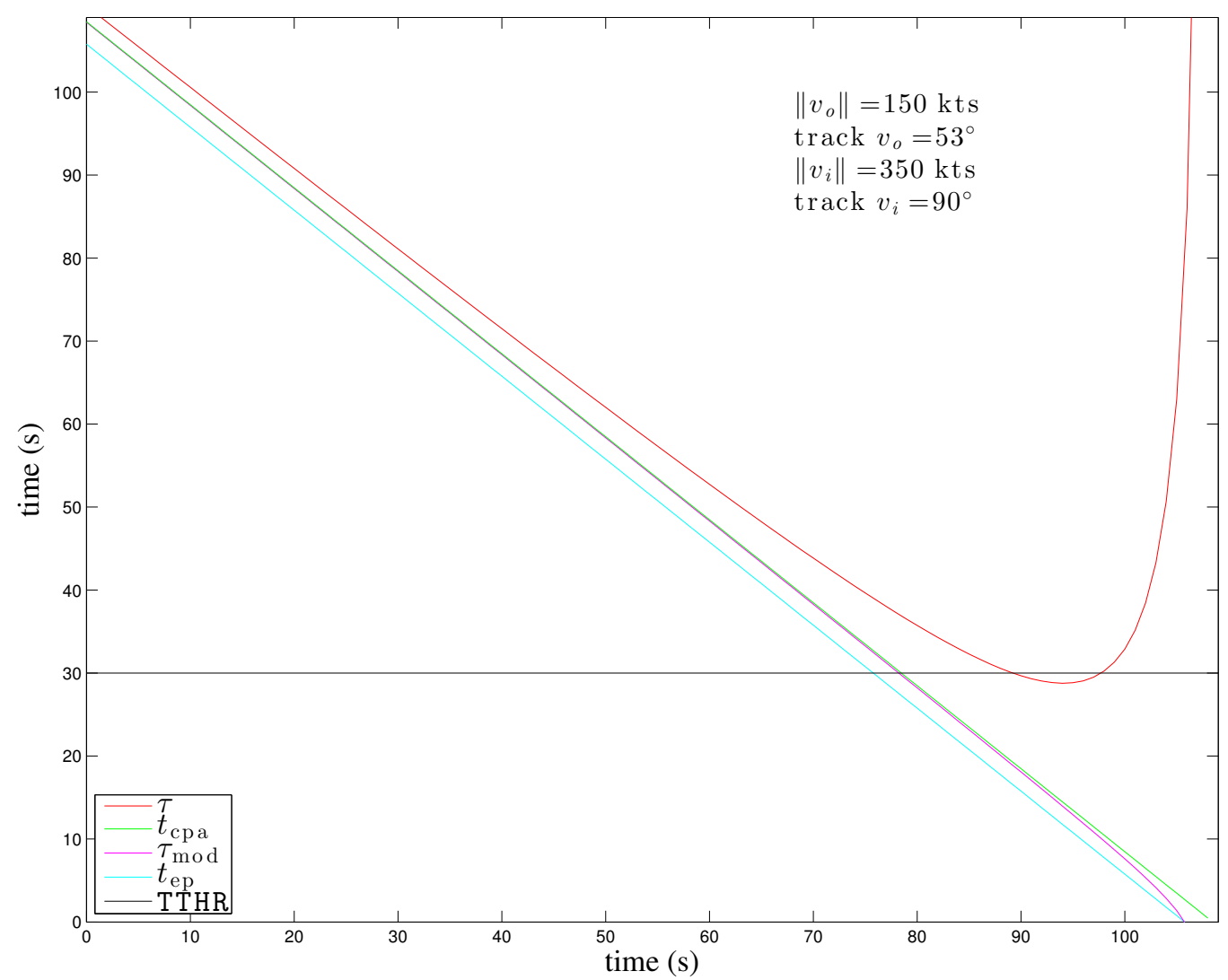

Figure 1: Time vs. $\tau, t_{\mathrm{cpa}}, \tau_{\mathrm{mod}}, t_{\mathrm{ep}}$

Theorem 2. Let $\mathbf{s}, \mathbf{v}$ be such that $\mathbf{s} \cdot \mathbf{v}<0,\|\mathbf{s}\|>D T H R$, and $d_{\text {cpa }}(\mathbf{s}, \mathbf{v}) \leq$ DTHR, i.e., the aircraft are horizontally converging, outside the distance threshold DTHR, and their distance at time of closest point of approach is less of equal than DTHR, the following inequalities hold

$$
t_{e p}(\mathbf{s}, \mathbf{v}) \leq \tau_{\text {mod }}(\mathbf{s}, \mathbf{v}) \leq t_{c p a}(\mathbf{s}, \mathbf{v}) \leq \tau(\mathbf{s}, \mathbf{v})
$$

${ }^{a}$ Aircraft headings are measured in true north clockwise convention, i.e., $0^{\circ}$ points to the north and degrees are positive in clockwise direction. 


\section{A Family of Well-Clear Boundary Models}

A well-clear boundary specifies the set of aircraft states that are considered to be in well-clear violation. Following the TCAS detection logic, the well-clear boundary models in this paper are specified by a logical condition that simultaneously checks horizontal and vertical violations. A horizontal violation occurs if the current range is less than a given horizontal distance threshold DTHR. A horizontal violation also occurs if distance at time of closest point of approach is less than DTHR and a given time variable $t_{\mathrm{var}}$ is less than a given time threshold TTHR. In the vertical dimension, a similar comparison is made. Vertical well clear is violated if the relative altitude is less than a given altitude threshold ZTHR or if the time to co-altitude is less than a given vertical time threshold TCOA. The distinct and altitude thresholds are considered to be positive numbers, i.e., DTHR $>0$ and ZTHR $>0$. The time thresholds are considered to be non-negative, i.e., TTHR $\geq 0$ and TCOA $\geq 0$. Formally, this well-clear violation condition can be denoted as follows.

$$
\begin{aligned}
W C V_{t_{\mathrm{var}}}\left(\mathbf{s}, s_{z}, \mathbf{v}, v_{z}\right) \equiv & \text { Horizontal_WCV }{ }_{t_{\mathrm{var}}}(\mathbf{s}, \mathbf{v}) \text { and } \\
& \operatorname{Vertical} \_\mathrm{WCV}\left(s_{z}, v_{z}\right),
\end{aligned}
$$

where

$$
\begin{aligned}
& \text { Horizontal_WCV } t_{\text {var }}(\mathbf{s}, \mathbf{v}) \equiv\|\mathbf{s}\| \leq \text { DTHR or } \\
& \left(d_{\mathrm{cpa}}(\mathbf{s}, \mathbf{v}) \leq \mathrm{DTHR} \text { and } 0 \leq t_{\mathrm{var}}(\mathbf{s}, \mathbf{v}) \leq \mathrm{TTHR}\right) \text {, } \\
& \text { Vertical_WCV }\left(s_{z}, v_{z}\right) \equiv\left|s_{z}\right| \leq \text { ZTHR or } 0 \leq t_{\text {coa }}\left(s_{z}, v_{z}\right) \leq \mathrm{TCOA} \text {. }
\end{aligned}
$$

The logical condition $W C V_{t_{\mathrm{var}}}$ defines a family of well-clear boundary models where $t_{\mathrm{var}}$ can be instantiated with any time variable and DTHR, TTHR, ZTHR, and TCOA are set to threshold values of interest. The fact that the time thresholds TTHR and TCOA can be zero allows for the definition of well-clear boundary models that do not depend on time thresholds. For instance, when TTHR $=0$ and TCOA $=0, W C V_{t_{\text {cpa }}}$ specifies the loss of separation condition for a cylindrical volume of radius DTHR and half-height ZTHR around one of the aircraft. Indeed, in this case, $W C V_{t_{\text {cpa }}}$ is logically equivalent to the logical condition $\|\mathbf{s}\| \leq \mathrm{DTHR}$ and $\left|s_{z}\right| \leq \mathrm{ZTHR}$.

The TCAS II RA core logic, provided in [5], is obtained by $W C V_{\tau_{\bmod }}$, where DTHR, TTHR, ZTHR, and TCOA are set to the TCAS II thresholds DMOD, TAU, ZTHR, and TAU, respectively. The actual values of these thresholds are given in a table indexed by sensitivity levels based on the ownship's altitude [8]. In the TCAS II RA logic, the logical condition $d_{\mathrm{cpa}}(\mathbf{s}, \mathbf{v}) \leq$ DTHR in the horizontal check is called horizontal miss-distance filter and, in that condition, DTHR is set to the miss-horizontal distance threshold HMD, which is equal to DMOD. The well-clear boundary model defined in [6] is obtained by $W C V_{t_{\mathrm{ep}}}$, where TCOA $=$ TTHR.

Henceforth, the well-clear models specified by $W C V_{\tau}, W C V_{\tau_{\text {mod }}}, W C V_{t_{\text {cpa }}}$, and $W C V_{t_{\text {ep }}}$ will be referred to as WC_TAU, WC_TAUMOD, WC_TCPA, and WC_TEP, respectively. The rest of this section studies properties and relations satisfied by these models.

\section{III.A. Symmetry}

A well-clear boundary model specified by $W C V_{t_{\mathrm{var}}}$, for a given time variable $t_{\mathrm{var}}$, is symmetric if and only if

$$
W C V_{t_{\mathrm{var}}}\left(\mathbf{s}, s_{z}, \mathbf{v}, v_{z}\right)=W C V_{t_{\mathrm{var}}}\left(-\mathbf{s},-s_{z},-\mathbf{v},-v_{z}\right) .
$$

In other words, in a symmetric well-clear boundary model both the ownship and intruder aircraft have the same perception of being well clear or not.

Theorem 3 (Symmetry). If $t_{v a r}$ is symmetric, the well-clear boundary model specified by $W C V_{t_{v a r}}$ is symmetric. Hence, by Theorem 1, the well-clear boundary models WC_TAU, WC_TAUMOD, WC_TCPA, and WC_TEP are symmetric for any choice of threshold values DTHR, TTHR, ZTHR, and TCOA.

\section{III.B. Inclusion}

Figures 2-5 illustrate the violation areas for the well-clear boundary models WC_TAU, WC_TAUMOD, WC_TCPA, and WC_TEP for the scenario of Figure 1. The threshold values used in this scenario are $\mathrm{DTHR}=1 \mathrm{nmi}$, TTHR $=\mathrm{TCOA}=30 \mathrm{~s}$, and ZTHR $=475 \mathrm{ft}$. The violation areas in these figures are similar to the conflict contours proposed in [9]. The points in these areas represent future locations of the ownship where a well-clear violation will occur assuming that the intruder aircraft continues its current trajectory and the 


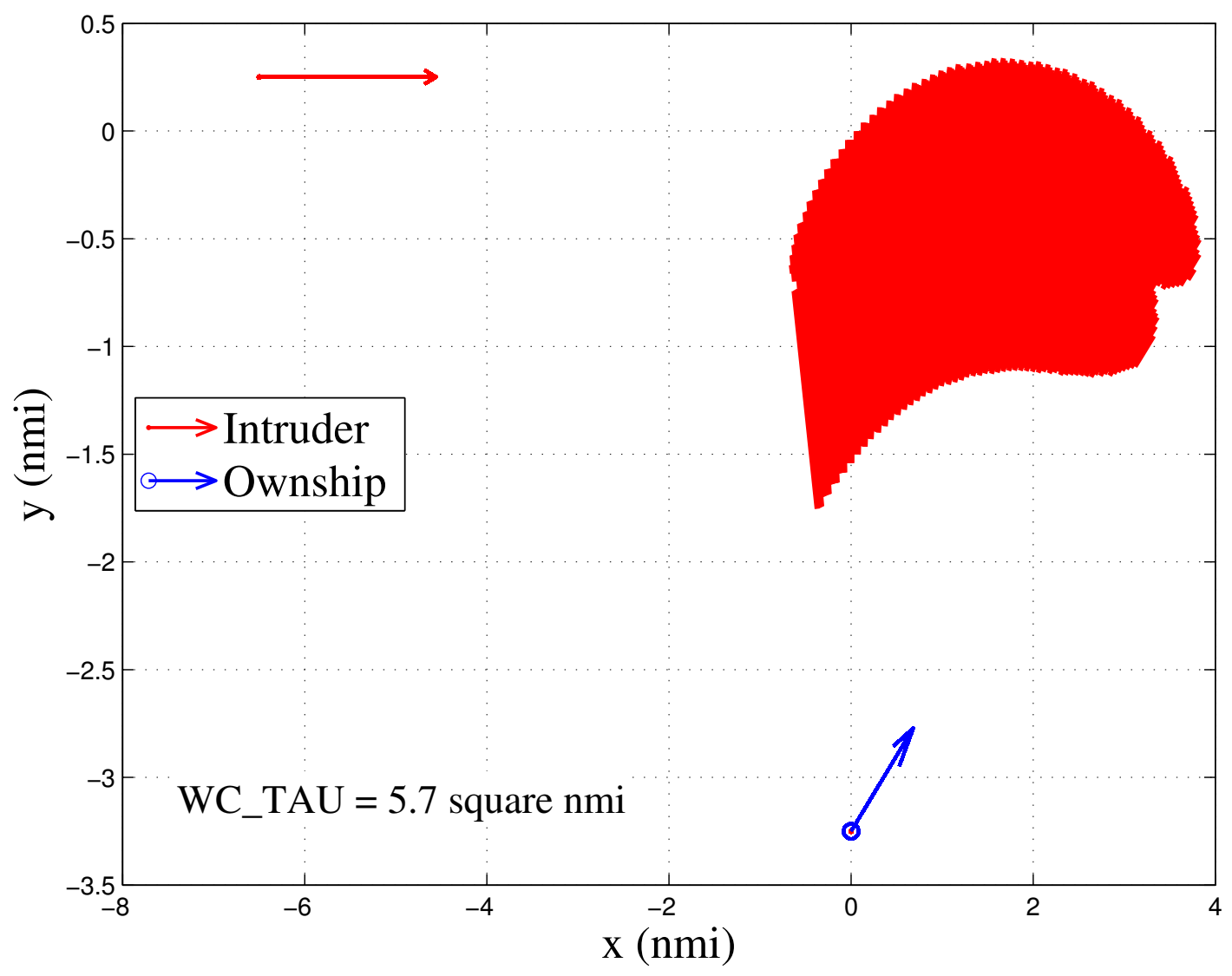

Figure 2: Violation areas of $W C V_{\tau}$ for scenario of Figure 1 


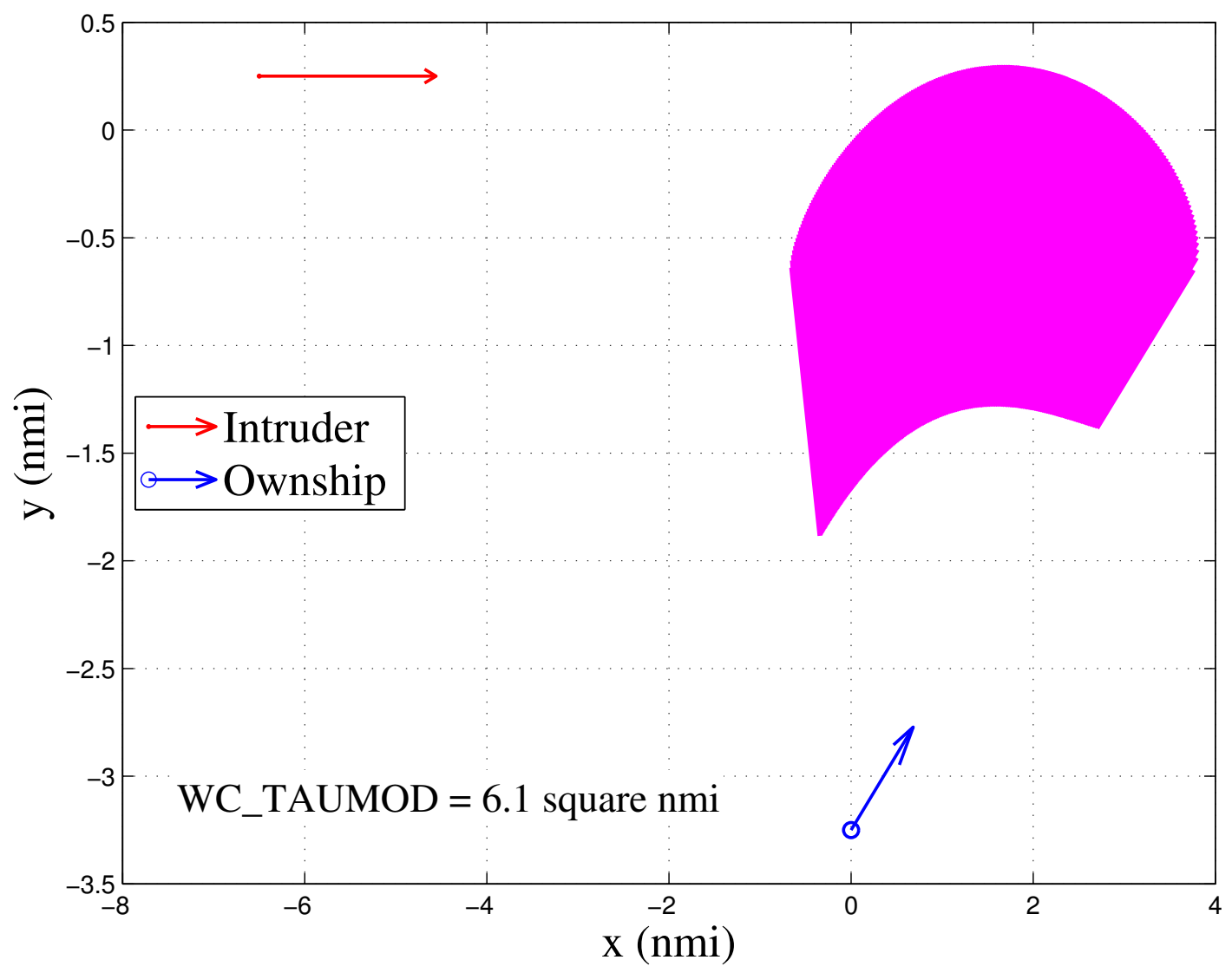

Figure 3: Violation areas of $W C V_{\tau_{\text {mod }}}$ for scenario of Figure 1 


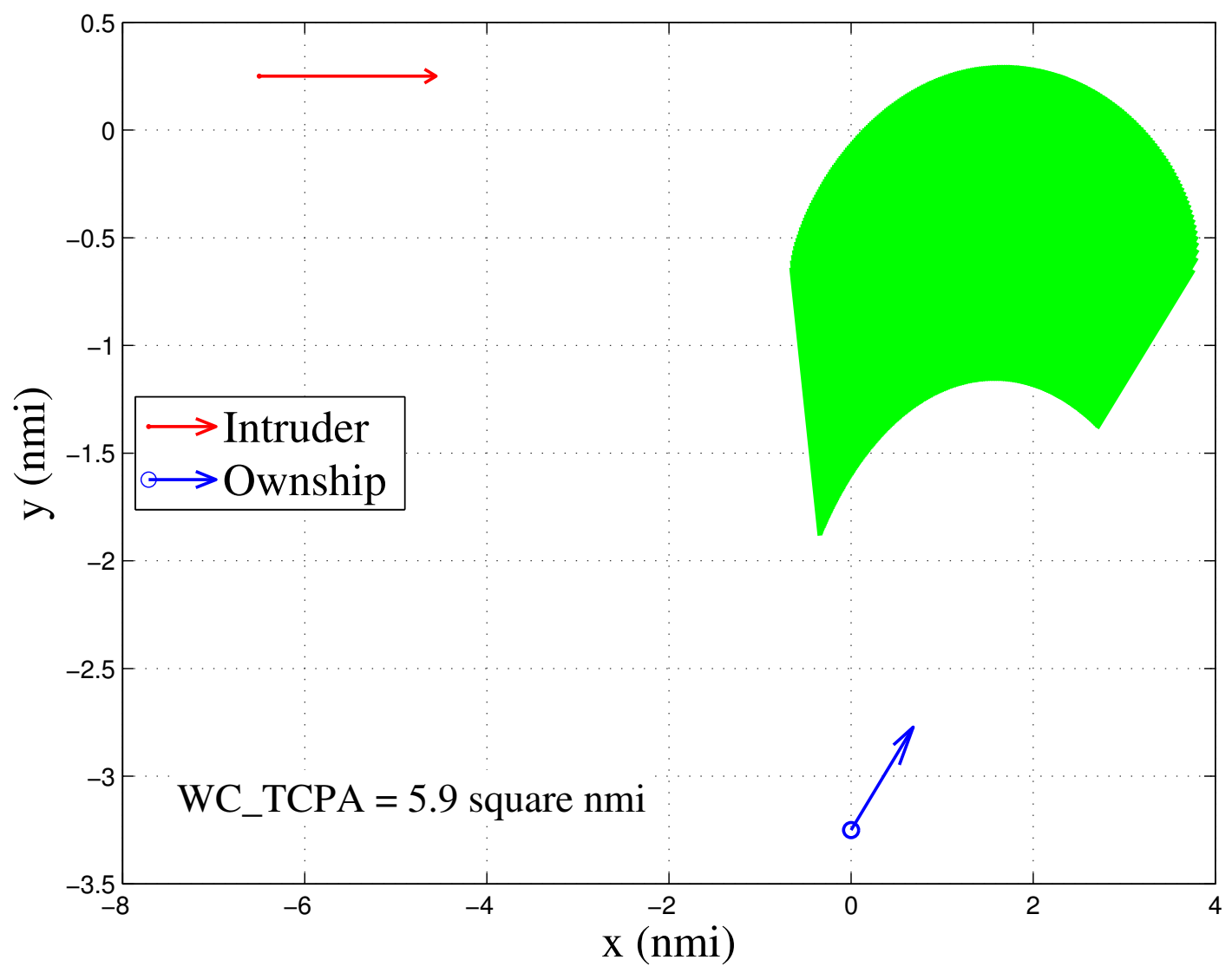

Figure 4: Violation areas of $W C V_{t_{\mathrm{cpa}}}$ for scenario of Figure 1 


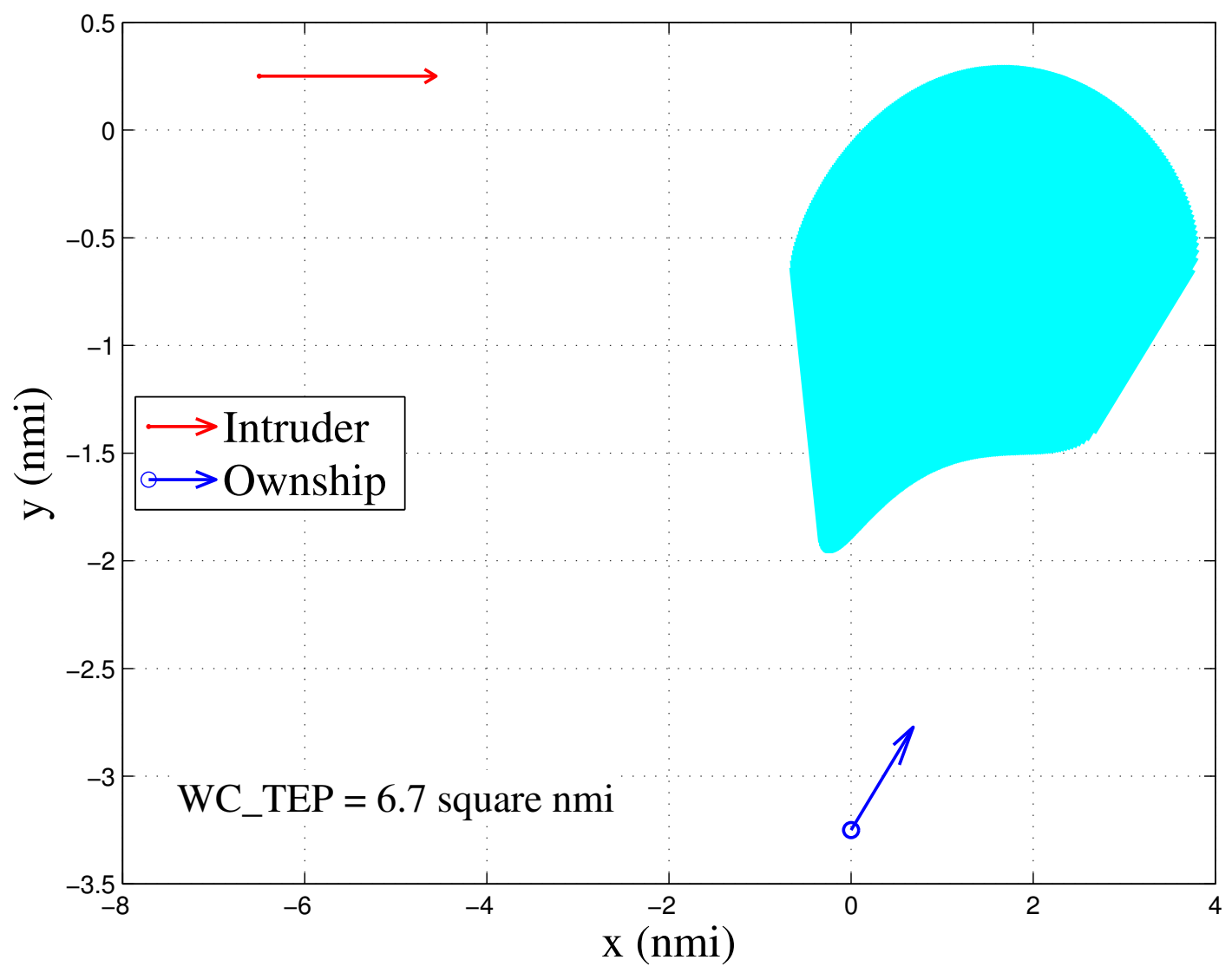

Figure 5: Violation areas of $W C V_{t_{\mathrm{ep}}}$ for scenario of Figure 1 


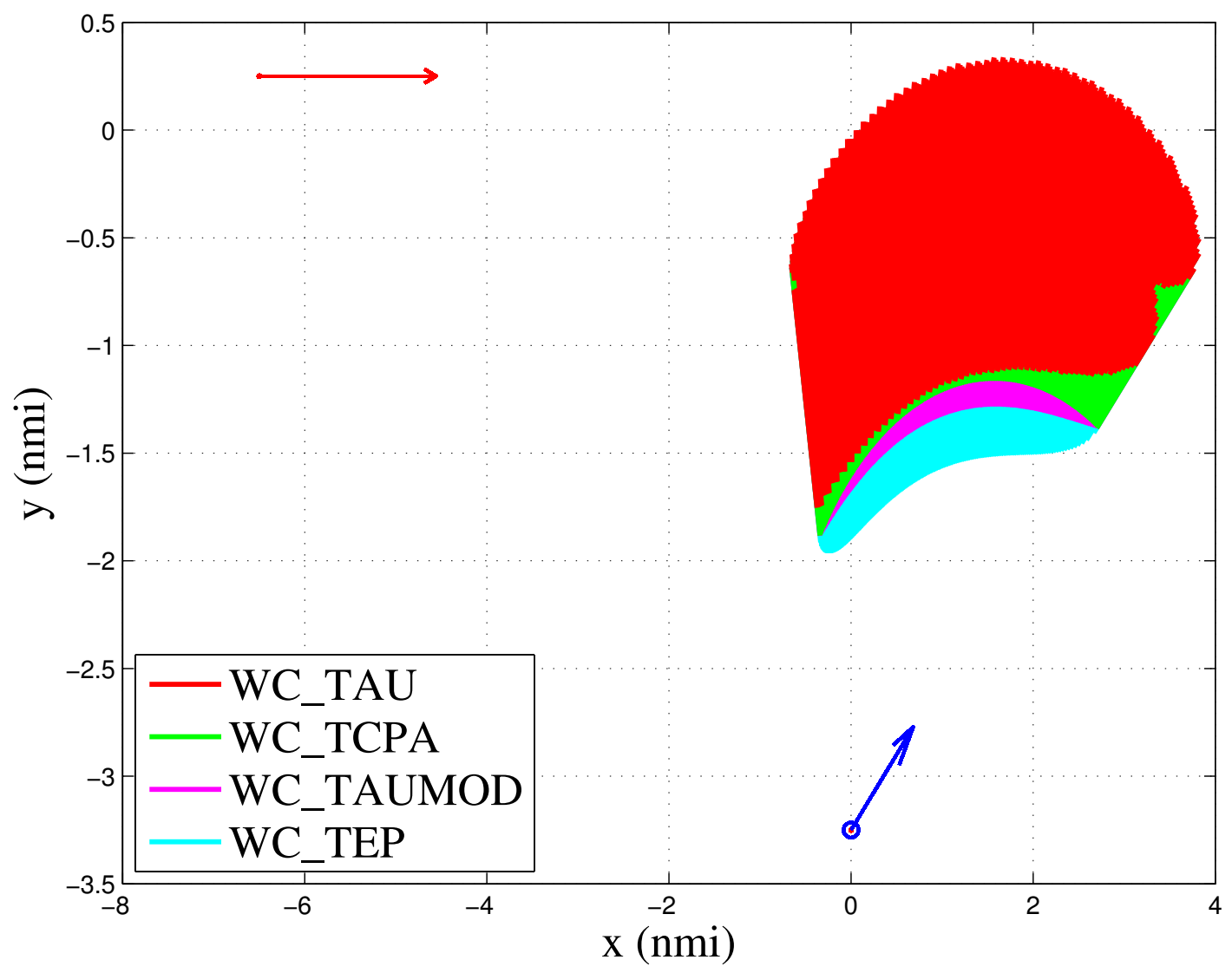

Figure 6: Overlay of violation areas for scenario of Figure 1 
ownship either continues its current trajectory or instantaneously changes its direction but keeps its ground speed.

Figure 6 overlays the violation areas for the four boundary models. This figure illustrates that for a common set of threshold values, the violation area of $\mathrm{WC}$ TAU is included in the violation area of WC_TCPA, which is included in the violation area of WC_TAUMOD, which is included in the violation area of WC_TEP. Theorem 4 below states that this inclusion property always holds for any encounter geometry and choice of common threshold values. Theorem 4 is a consequence of Theorem 2 .

Theorem 4 (Inclusion). For all $\mathbf{s}, s_{z}, \mathbf{v}, v_{z}$ and choice of threshold values DTHR, TTHR, ZTHR, and TCOA, the following implications hold

(i) $W C V_{\tau}\left(\mathbf{s}, s_{z}, \mathbf{v}, v_{z}\right) \Longrightarrow W C V_{t_{c p a}}\left(\mathbf{s}, s_{z}, \mathbf{v}, v_{z}\right)$,

(ii) $W C V_{t_{c p a}}\left(\mathbf{s}, s_{z}, \mathbf{v}, v_{z}\right) \Longrightarrow W C V_{\tau_{m o d}}\left(\mathbf{s}, s_{z}, \mathbf{v}, v_{z}\right)$, and

(iii) $W C V_{\tau_{\text {mod }}}\left(\mathbf{s}, s_{z}, \mathbf{v}, v_{z}\right) \Longrightarrow W C V_{t_{e p}}\left(\mathbf{s}, s_{z}, \mathbf{v}, v_{z}\right)$.

A key consequence of Theorem 4 is that of the four well-clear boundary models, WC_TEP provides the most conservative safety margins in terms of having the largest violation area and the earliest time whereby a well-clear violation is defined to occur. The remaining models can be ordered from most conservative to least conservative as WC_TAUMOD, WC_TCPA, and WC_TAU.

\section{III.C. Local Convexity}

As illustrated by Figures 2-5, the violation areas are not geometrically convex. However, Figures 3-5 show that from the point of view of the ownship, any ray that points towards the violation area has only one intersecting segment. This property is referred to as local convexity. It can be verified by inspection of Figure 2 that this property does not always hold in the case of WC_TAU. A formal definition of local convexity follows.

Definition 1 (Local convexity). A well-clear boundary model specified by $W C V_{t_{v a r}}$, for a given time variable $t_{v a r}$, is locally convex if and only if there are no times $0 \leq t_{1} \leq t_{2} \leq t_{3} \leq T$ such that

1. the aircraft are not well clear at time $t_{1}$, i.e., $W C V_{t_{v a r}}\left(\mathbf{s}+t_{1} \mathbf{v}, s_{z}+t_{1} v_{z}, \mathbf{v}, v_{z}\right)$,

2. the aircraft are well clear at time $t_{2}$, i.e., $\neg W C V_{t_{v a r}}\left(\mathbf{s}+t_{2} \mathbf{v}, s_{z}+t_{2} v_{z}, \mathbf{v}, v_{z}\right)$, and

3. the aircraft not well clear at time $t_{3}$, i.e., $W C V_{t_{v a r}}\left(\mathbf{s}+t_{3} \mathbf{v}, s_{z}+t_{3} v_{z}, \mathbf{v}, v_{z}\right)$.

Thus, a well-clear boundary model is locally convex if for any ownship straight-line trajectory there is at most one time interval where the aircraft are not well clear.

Theorem 5. For any choice of threshold values, the well-clear boundary models WC_TCPA, WC_TAUMOD, and $W C_{\text {_TEP }}$ are locally convex.

As illustrated by Figure 2, the well-clear boundary model WC_TAU is not locally convex for all choices of threshold values. In particular, it can be seen in Figure 1, assuming straight-line trajectories, that for the same encounter scenario the aircraft will have a well-clear violation at $91 \mathrm{~s}, 7$ seconds later they will be well clear, and 7 seconds after being well clear, they will have another well-clear violation.

Theorem 6. For some choices of threshold values, the well-clear boundary model WC_TAU is not locally convex.

\section{Well-Clear Violation Detection Algorithms}

A conflict detection algorithm checks whether or not a loss of separation is predicted to occur within a given period of time. Similarly, it is possible to design detection algorithms of well-clear violations for a given well-clear model. This section presents analytical formulations of such well-clear violation detection algoritms for WC_TAUMOD, WC_TCPA, and WC_TEP. In particular, the functions detection_WCV $\tau_{\tau_{\text {mod }}}$, detection_ $W_{C V} t_{t_{\text {cpa }}}$ and detection_ $W_{C V} t_{\text {ep }}$ are defined. These functions take as inputs the relative horizontal 
and vertical states of the aircraft and a lookahead time interval $[B, T]$, with $0 \leq B<T$, and return an interval of violation for $W C V_{\tau_{\text {mod }}}, W C V_{t_{\mathrm{cpa}}}$, and $W C V_{t_{\mathrm{ep}}}$, respectively. Assuming accurate vector information and constant velocities, it has been formally proved that these functions correctly and completely characterize the aircraft states that lead to a well-clear violation, within the lookahead time interval, for their respective models,

To define an algorithm detection_WCV $t_{t_{\mathrm{var}}}$ for a given time variable $t_{\mathrm{var}}$, it is necessary to define functions that detect time intervals of violation for the horizontal and vertical dimension. Since the vertical check Vertical_WCV (Formula (13)) is independent of $t_{\mathrm{var}}$, the algorithm that computes the time interval for a vertical well-clear violation is the same for any definition in the family of $W C V_{t_{\mathrm{var}}}$ models. Formula (15) specifies an algorithm for detecting such vertical well-clear violations. The function detection_VWCV has as inputs a relative vertical state and a lookahead time interval $[B, T]$. It returns a time interval within $[B, T]$ when a vertical well-clear violation will occur. The interval is empty if no such violation occurs.

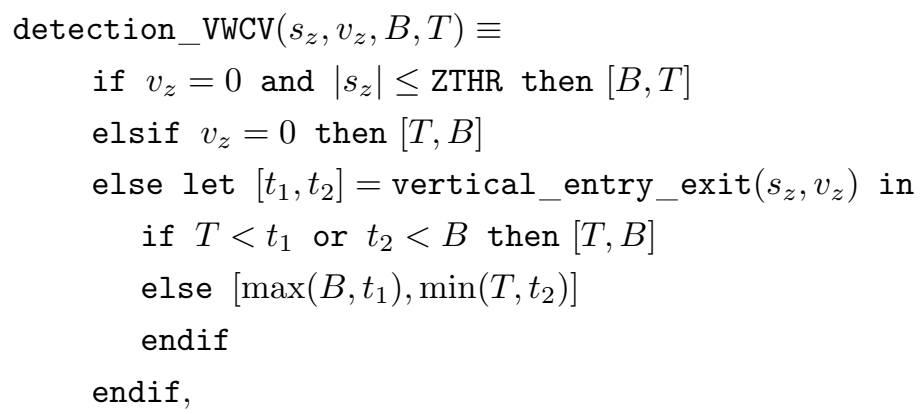

where

$$
\begin{aligned}
& \text { vertical_entry_exit }\left(s_{z}, v_{z}\right) \equiv \\
& \text { let } H=\max (\mathrm{ZTHR}, \mathrm{TCOA}|v z|) \text { in } \\
& {\left[\frac{-\operatorname{sign}\left(v_{z}\right) H-s_{z}}{v_{z}}, \frac{\operatorname{sign}\left(v_{z}\right) \mathrm{ZTHR}-s_{z}}{v_{z}}\right],}
\end{aligned}
$$

The horizontal check Horizontal__WCV $t_{\text {var }}$ (Formula (13)) depends on actual definition of the time variable $t_{\mathrm{var}}$. Hence, a horizontal detection algorithm for each particular definition of $t_{\mathrm{var}}$ is necessary. Formula (17), Formula (18), and Formula (19) specify horizontal detection algorithms for WC_TAUMOD, WC_TCPA, and WC_TEP, respectively. The functions specified by these formulas have as inputs a relative horizontal state and a lookahead time $T$. They return a time interval within $[B, T]$ when a horizontal well-clear violation for their respective model will occur. The interval is empty if no such violation occurs.

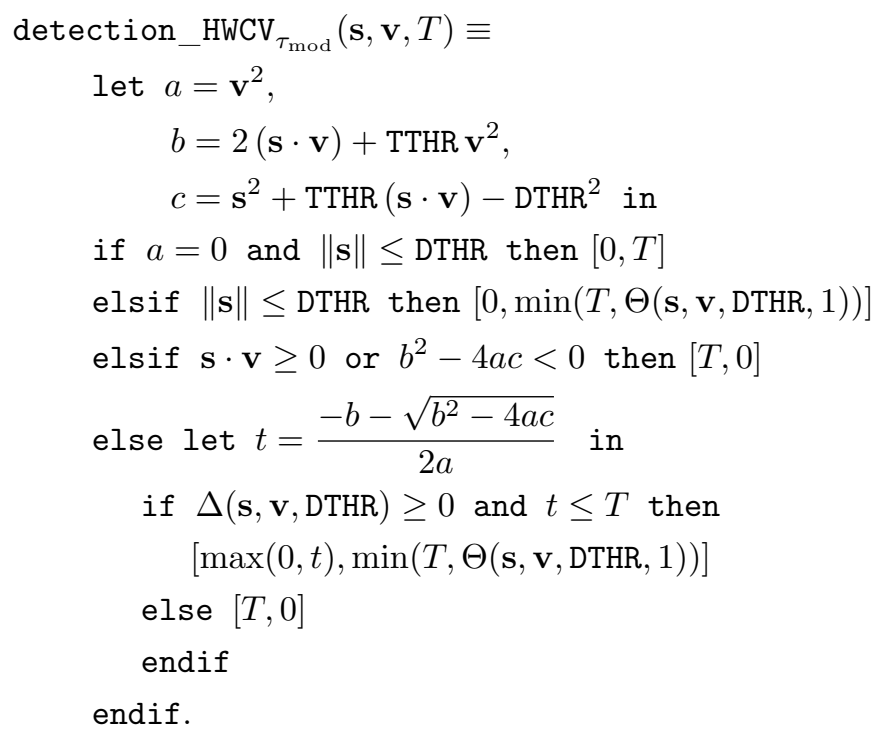




$$
\begin{aligned}
& \text { detection_HWCV } t_{\text {cpa }}(\mathbf{s}, \mathbf{v}, T) \equiv \\
& \text { if }\|\mathbf{v}\|=0 \text { and }\|\mathbf{s}\| \leq \operatorname{DTHR} \text { then }[0, T] \\
& \text { elsif }\|\mathbf{v}\|=0 \text { then }[T, 0] \\
& \text { elsif }\|\mathbf{s}\| \leq \text { DTHR then }[0, \min (T, \Theta(\mathbf{s}, \mathbf{v}, \mathrm{DTHR}, 1))] \\
& \text { elsif } \mathbf{s} \cdot \mathbf{v}>0 \text { then }[T, 0] \\
& \text { elsif }\left\|\mathbf{s}+t_{\text {cpa }}(\mathbf{s}, \mathbf{v}) \mathbf{v}\right\|>\text { DTHR then }[T, 0] \\
& \text { elsif } \Delta(\mathbf{s}, \mathbf{v}, \text { DTHR })<0 \text { and } t_{\mathrm{cpa}}(\mathbf{s}, \mathbf{v})-\text { TTHR }>T \text { then }[T, 0] \\
& \text { elsif } \Delta(\mathbf{s}, \mathbf{v}, \text { DTHR })<0 \text { then } \\
& {\left[\max \left(0, t_{\mathrm{cpa}}(\mathbf{s}, \mathbf{v})-\mathrm{TTHR}\right), \min \left(T, t_{\mathrm{cpa}}(\mathbf{s}, \mathbf{v})\right)\right]} \\
& \text { else let } t_{\min }=\min \left(\Theta(\mathbf{s}, \mathbf{v}, \text { DTHR, }-1), t_{\mathrm{cpa}}(\mathbf{s}, \mathbf{v})-\mathrm{TTHR}\right) \text { in } \\
& \text { if } t_{\min }>T \text { then }[T, 0] \\
& \text { else }\left[\max \left(0, t_{\min }\right), \min (T, \Theta(\mathbf{s}, \mathbf{v}, \text { DTHR }, 1))\right] \\
& \text { endif } \\
& \text { endif. } \\
& \text { detection_HWCV }{ }_{t_{\mathrm{ep}}}(\mathbf{s}, \mathbf{v}, T) \equiv \\
& \text { if }\|\mathbf{v}\|=0 \text { and }\|\mathbf{s}\| \leq \text { DTHR then }[0, T] \\
& \text { elsif }\|\mathbf{v}\|=0 \text { then }[T, 0] \\
& \text { elsif }\|\mathbf{s}\| \leq \text { DTHR then }[0, \min (T, \Theta(\mathbf{s}, \mathbf{v}, \text { DTHR }, 1))] \\
& \text { elsif } \mathbf{s} \cdot \mathbf{v}>0 \text { then }[T, 0] \\
& \text { elsif } \Delta(\mathbf{s}, \mathbf{v}, \text { DTHR })<0 \text { or } \Theta((\mathbf{s}, \mathbf{v}, \text { DTHR, }-1)-\text { TTHR }>T \text { then }[T, 0] \\
& \text { else }[(\max (0, \Theta(\mathbf{s}, \mathbf{v}, \text { DTHR, }-1)-\mathrm{TTHR}), \min (T, \Theta(\mathbf{s}, \mathbf{v}, \mathrm{DTHR}, 1))] \\
& \text { endif. }
\end{aligned}
$$

Given a definition of Horizontal_WCV $t_{\text {var }}$, Formula 20 specifies a function that computes a time interval of violation for $W C V_{t_{\mathrm{var}}}$. The function detection_ $\mathrm{WCV}_{t_{\mathrm{var}}}$ has as inputs a relative state and a lookahead time interval $[B, T]$. It returns a time interval $\left[t_{\text {in }}, t_{\text {out }}\right]$ when a violation of $W C V_{t_{\text {var }}}$ will occur. The interval is empty if no such violation occurs. Theorem 7 states that detection ${ } \mathrm{WCV}_{t_{\mathrm{var}}}$ completely characterizes the relative aircraft states that lead to a well-clear violation of $W C V_{t_{\mathrm{var}}}$, for $t_{\mathrm{var}}$ one of $\tau_{\mathrm{mod}}, t_{\mathrm{cpa}}$, or $t_{\mathrm{ep}}$, within a lookahead time interval $[B, T]$ and assuming straight-line trajectories for both aircraft.

$$
\begin{aligned}
& \text { detection_WCV }{ }_{t_{\text {var }}}\left(\mathbf{s}, s_{z}, \mathbf{v}, v_{z}, B, T\right) \equiv \\
& \text { let }\left[t_{1}, t_{2}\right]=\text { detection_VWCV }\left(s_{z}, v_{z}, B, T\right) \text { in } \\
& \text { if } t_{1}>t_{2} \text { then }[T, B] \\
& \text { elsif } t_{1}=t_{2} \text { and Horizontal_WCV } t_{\mathrm{var}}\left(\mathbf{s}+t_{1} \mathbf{v}, \mathbf{v}\right) \text { then }\left[t_{1}, t_{1}\right] \\
& \text { elsif } t_{1}=t_{2} \text { then }[T, B] \\
& \text { else let }\left[t_{\text {in }}, t_{\text {out }}\right]=\text { detection_HWCV } t_{t_{\text {var }}}\left(\mathbf{s}+t_{1} \mathbf{v}, \mathbf{v}, t_{2}-t_{1}\right) \text { in } \\
& {\left[t_{\text {in }}+t_{1}, t_{\text {out }}+t_{1}\right]} \\
& \text { endif. }
\end{aligned}
$$

Theorem 7. Let $t_{v a r}$ be one of $\tau_{m o d}, t_{c p a}$, or $t_{e p}$. The function detection_WCV $t_{t_{v a r}}$ is a correct and complete detection algorithm for $W C V_{t_{v a r}}$, i.e., for all relative states $\mathbf{s}, s_{z}, \mathbf{v}, v_{z}$, time interval $[B, T]$, with $0 \leq B<T$, and $t \in[B, T], W C V_{t_{v a r}}\left(\mathbf{s}+t \mathbf{v}, s_{z}+t v_{z}, \mathbf{v}, v_{z}\right)$ holds if and only if $t \in\left[t_{i n}, t_{\text {out }}\right]$, where

$$
\left[t_{\text {in }}, t_{\text {out }}\right]=\text { detection_WCV } \operatorname{tvar}_{t_{\text {ar }}}\left(\mathbf{s}, s_{z}, \mathbf{v}, v_{z}, B, T\right) .
$$




\section{Conclusion}

A family of well-clear boundary models is presented. This family generalizes the TCAS II Resolution Logic with different possible definition of horizontal time variables including tau, modified tau, time to closest point of approach, and time to entry point. Analytical techniques are used to study the properties of this model. For instance, it has been formally proved that the well-clear model based on time to entry point is more conservative than tau, modified tau, and time to closest point of approach for any scenario and any common choice of threshold values. Furthermore, it is shown that all the models in this family are symmetric, i.e., the ownship and intruder aircraft have the same perception of being well-clear or not at any moment in time. Except for the model based on tau, all the models are locally convex meaning that there is at most one interval of time when the aircraft are not well-clear, assuming straight-line trajectories.

Detection algorithms for a family of well-clear boundary models are proposed which compute the time interval of violation within a given lookahead time interval. These algorithms could be used in the implementation of airborne and ground capabilities that allow UAS pilots to avoid encounter scenarios that are not well clear.

The mathematical development presented in this paper has been mechanically verified in the Prototype Verification System (PVS) [7]. This level of rigor is justified by the safety-critical nature of the well-clear concept in the integration of Unmanned Aerial Vehicles into the National Aerospace System.

\section{References}

\footnotetext{
${ }^{1}$ María Consiglio, James Chamberlain, César Muñoz, and Keith Hoffler. Concept of integration for UAS operations in the NAS. In Proceedings of 28th International Congress of the Aeronautical Sciences, ICAS 2012, Brisbane, Australia, 2012.

${ }^{2}$ FAA Sponsored Sense and Avoid Workshop. Sense and avoid (SAA) for Unmanned Aircraft Systems (UAS), October 2009.

${ }^{3}$ Jonathan Hammer. Horizontal miss distance filter system for suppressing false resolution alerts, October 1996. U.S. Patent 5,566,074.

${ }^{4}$ James Kuchar and Lee Yang. A review of conflict detection and resolution modeling methods. IEEE Transactions on Intelligent Transportation Systems, 1(4):179-189, December 2000.

${ }^{5}$ César Muñoz, Anthony Narkawicz, and James Chamberlain. A TCAS-II resolution advisory detection algorithm. In Proceedings of the AIAA Guidance Navigation, and Control Conference and Exhibit 2013, AIAA-2013-4622, Boston, Massachusetts, 2013.

${ }^{6}$ Anthony J. Narkawicz, César A. Muñoz, Jason M. Upchurch, James P. Chamberlain, and María C. Consiglio. A wellclear volume based on time to entry point. Technical Memorandum NASA/TM-2014-218155, NASA, Langley Research Center, Hampton VA 23681-2199, USA, January 2014.

${ }^{7}$ S. Owre, J. Rushby, and N. Shankar. PVS: A prototype verification system. In Deepak Kapur, editor, Proc. 11th Int. Conf. on Automated Deduction, volume 607 of Lecture Notes in Artificial Intelligence, pages 748-752. Springer-Verlag, June 1992.

${ }^{8}$ RTCA SC-147. RTCA-DO-185B, Minimum operational performance standards for traffic alert and collision avoidance system II (TCAS II), July 2009.

${ }^{9}$ J. Tadema, E. Theunissen, and K.M. Kirk. Self separation support for UAS. In AIAA Infotech@Aerospace 2010, number AIAA-2010-3460, Atlanta, GA, USA, April 2010.

${ }^{10}$ U.S. Department of Transportation Federal Aviation Adminstration. Introduction to TCAS II Version 7.1, February 2011.
} 